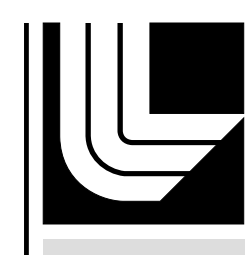

LAW RENCE LIVERMORE N A T IO N A L LABORATORY

\title{
A Steinberg-Guinan model for High-Pressure Carbon, Diamond Phase
}

D. Orlikowski, A. Correa, E. Schwegler, J. Klepeis

July 30, 2007

Shock Compression of Condensed Matter

Kona, HI, United States

June 25, 2007 through June 29, 2007 
This document was prepared as an account of work sponsored by an agency of the United States Government. Neither the United States Government nor the University of California nor any of their employees, makes any warranty, express or implied, or assumes any legal liability or responsibility for the accuracy, completeness, or usefulness of any information, apparatus, product, or process disclosed, or represents that its use would not infringe privately owned rights. Reference herein to any specific commercial product, process, or service by trade name, trademark, manufacturer, or otherwise, does not necessarily constitute or imply its endorsement, recommendation, or favoring by the United States Government or the University of California. The views and opinions of authors expressed herein do not necessarily state or reflect those of the United States Government or the University of California, and shall not be used for advertising or product endorsement purposes. 


\title{
A Steinberg-Guinan model for High-Pressure Carbon, Diamond Phase
}

\author{
Daniel Orlikowski*, Alfredo A. Correa*, Eric Schwegler* and John E. Klepeis* \\ ${ }^{*}$ LLNL, P.O. Box 808, L-45, Livermore, CA 94551-0808
}

\begin{abstract}
Since the carbon, diamond phase has such a high yield strength, dynamic simulations must account for strength even for strong shock waves ( 3 Mbar). We have determined an initial parametrization of two strength models: Steinberg-Guinan (SG) and a modified or improved SG, that captures the high pressure dependence of the calculated shear modulus up to $10 \mathrm{Mbar}$. The models are based upon available experimental data and on calculated elastic moduli using robust density functional theory. Additionally, we have evaluated these models using hydrodynamic simulations of planar shocks experiments.
\end{abstract}

Keywords: strength, carbon, diamond, continuum model

PACS: 62.20.Fe,81.05.Uw,62.50.+p,31.15.Ew

\section{INTRODUCTION}

With the pursuit of obtaining experimental pressures beyond $15 \mathrm{Mbar}$, there is significant interest and effort in characterizing not only the objective material, like hydrogen, but also all the support materials, which must have an equal level of characterizing and modeling. One such material is the carbon diamond phase, that is experimentally stable up to $3 \mathrm{Mbar}[1]$ and theoretically stable to $\sim 10 \mathrm{Mbar}$ at ambient temperature[2]. Since diamond is expected to have a high yield strength $[3,4,5]$, it cannot be ignored in the modeling and design-work of future experiments, even to very high pressures $(\approx 3 \mathrm{Mbar})$. We intend here to initiate a parametrization for a high-pressure, strength model, specific to the carbon diamond phase.

The strength of diamond has been investigated but never conclusively determined at ambient pressure $Y_{0}=0.35 \mathrm{Mbar}[3]$, but at pressures near $2 \mathrm{Mbar}$ $Y_{0}=1.3-1.4 \mathrm{Mbar}$ has been experimentally determined at $T=300 \mathrm{~K}[4]$. The theoretical calculations also have been performed giving an upper bound of $Y_{0}=0.95-2.0 \mathrm{Mbar}$ for $P=0$ to $3 \mathrm{Mbar}[6,5]$. Furthermore, other considerations must be understood for dynamic simulations. Little is known about the high-pressure mechanism that underlies the observed two wave structure in shock wave experiments, i.e. brittle or ductile response. Additionally, a temperature transition from brittle to ductile response is expected near $2000 \mathrm{~K}$ at ambient pressure[7]. From calculations a transformation to a graphite-like phase has been shown for high shear stresses[8]. And considering that under high confinement and under a slow strain-rate, a transition from brittle to ductile behavior may occur[9], thus complicating the modeling efforts, at this time. Therefore, in light of the these unknowns, currently, the most accommodating model is the Steinberg-Guinan model.

This work is part of a larger effort that is presented in these proceedings towards the understanding of the high-pressure response of carbon in general, due to either shock or ramp wave loading. We present the methodology of determining two strength models, and have performed hydrodynamic simulations to evaluate the parametrization as compared to available free surface particle velocity histories.

\section{METHODOLOGY \& SIMULATIONS}

The parametrization of two strength models is given - the Steinberg-Guinan (SG)[11] and the improved SG (ISG) models. This is based upon 
limited experimental evidence and upon robust first-principle calculations of the elastic moduli for diamond. In these models we ignore strain-rate effects and focus on the shear modulus and the yield.

For the constitutive modeling, the longitudinal stress as given by the von Mises criterion is $\sigma(P, T)=P+\frac{2}{3} Y(P, T)$. The SG model describes the yield strength $Y$ as a scaled quantity by the shear modulus

$$
\begin{array}{r}
Y(P, T) \propto Y_{0}\left[G(P, T) / G_{0}\right] \\
G(P, T)=G_{0}\left\{1+A_{0} P(\eta)^{-1 / 3}+B_{0}(T-300)\right\}
\end{array}
$$

and $G_{0}=G(P=0, T=300 \mathrm{~K})$ and where $\eta=$ $\rho / \rho_{0}$.[11] The two constants $A_{0}=1 / G_{0}(d G / d P)$ and $B_{0}=1 / G_{0}(d G / d T)$ are evaluated at ambient conditions $P=0$ and $T=300 \mathrm{~K}$. The SG model ignores strain-rate effects.

In considering the pressure dependence of the SG model with respect to the DFT based calculations of $G(P, T \sim 0 K)$ in Fig. 1, a second model, an improved SG (ISG) model, is described here. To agree with the pressure dependence of calculated $G(P, T \approx 300 K)$, a function $f(\eta)=1+\exp \left[\alpha_{i s g}\left(\eta-\eta_{i s g}\right)\right]$ is introduced that softens the high-pressure dependence of the SG model to one that is more linear in nature and is written as follows:

$$
\begin{array}{r}
G(P, T)=G_{0}\left\{\left[1+A_{0} P(\eta)^{-1 / 3}\right] f(\eta)\right. \\
\left.+(1-f(\eta))\left[A_{i s g}+M_{i s g} \eta\right]+B_{0}(T-300)\right\} .
\end{array}
$$

The constants $\alpha_{i s g}, \eta_{i s g}, A_{i s g}$ and $M_{i s g}$ are determined by fitting to the calculated $G(P, T \sim 0 K)$.

The shear modulus, either Eqn. 2 or 3 , is parametrized in two separate ways through ambient experimental values and through calculated singlecrystal, elastic moduli over a range of pressure, using robust density functional theory (DFT) $[13,14]$ via ABINIT plane-wave code[15]. The presented calculations are similar to the equation-of-state (EOS) calculations used in determining the high temperature and pressure phase diagram[2]. The DFT calculations were performed using norm-conserving pseudo-potential[16] with the generalized gradient approximation (GGA).[17] A $10 \times 10 \times 10$ $k$-point Monkhorst-Pack[18] mesh was used to sample the irreducible Brillouin zone. The energy cut off was determined to be $35 \mathrm{Ha}$. For the elastic moduli $C^{\prime}=\frac{1}{2}\left(C_{11}-C_{12}\right)$ and $C_{44}$, tetragonal and
TABLE 1. A comparison of available elastic moduli data at ambient conditions and $T \sim 0 \mathrm{~K}$ for the calculations (bottom 2 lines). The variation between calculations are due to differences in the determined lattice parameters used (6.729 a.u. (here) versus 6.743 a.u. [19])..

\begin{tabular}{lccc}
\hline & $C_{11}$ & $C_{12}$ & $C_{44}$ (Mbar) \\
\hline McSkimin[21] & 10.79 & 1.24 & 5.78 \\
Grimsditch[22] & 10.76 & 1.25 & 5.77 \\
\hline Mounet (GGA)[19] & 10.6 & 1.25 & 5.62 \\
Correa (GGA) & 10.4 & 0.89 & 5.65 \\
\hline
\end{tabular}

orthorhombic distortions (respectively) to the unit cell are performed with finite displacements less than 0.01 a.u. for 21 volumes spanning 42.88 to 15.63 a.u. The elastic moduli at $\mathrm{P}=0$ are compared in Table 1.

To obtain an aggregate polycrystalline $G(P, T)$, we have used the Voigt average $G_{V}=\frac{1}{5}\left(C_{11}-C_{12}+\right.$ $\left.3 C_{44}\right)$. The DFT calculated $G_{V}(P, T \sim 300)$ is compared in Fig. 1 to a parametrization of the SG model based on the DFT calculations at ambient pressure, demonstrating the significant disagreement of the SG model with increasing $\mathrm{P}$. This is expected since it would be quite remarkable to have ambient quantities describe material properties at $>50 \%$ compression.

For the temperature dependence of the elastic moduli, there are developed methods that are either within the quasi-harmonic limit or that completely capture the anharmonic effects[20]. However, partly due to the computational effort and partly to little understanding beyond the Hugoniot elastic limit(HEL), we use experimentally determined temperature derivative at ambient pressure to parametrize the models. Using McSkimin[21] values with Voigt averaging gives $A_{0}=0.44 \mathrm{Mbar}^{-1}$, $B_{0}=0.15 e-4 \mathrm{~K}^{-1}$, and $G_{0}=5.378 \mathrm{Mbar}$. The same parameters based on the DFT elastic moduli are $A_{0}=0.43 \mathrm{Mbar}^{-1}$, and $G_{0}=5.29$ Mbar agreeing to $\sim 2 \%$ (there is no temperature dependence calculated as yet). Using Eqn. 3 to fit the DFT shear modulus in Fig. 1 gives a parameter set of $A_{0}=-0.43 \mathrm{Mbar}^{-1}$, $\alpha_{i s g}=3.47, \eta_{i s g}=0.88, A_{i s g}=-0.596$, and $M_{i s g}=$ 1.588 .

To calculate the yield $Y(P, T)$, we follow the SG model Eqn. 1. For the $Y_{0}$, we can only give a lower bracket and then make some adjustments in compar- 


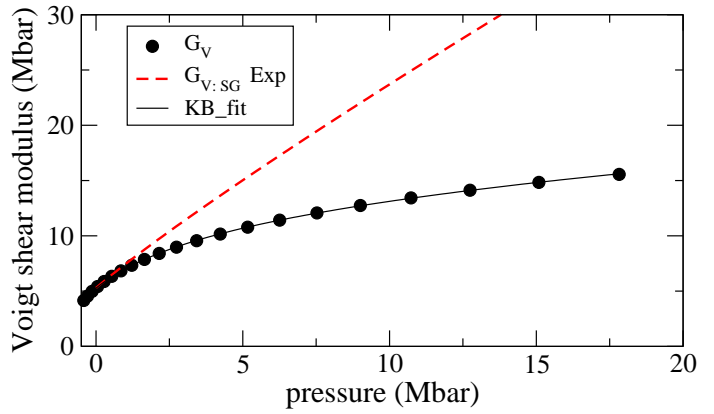

FIGURE 1. The pressure dependence only of the shear modulus for the parametrized SG model based upon DFT calculations at $\mathrm{P}=0$ ( dashed line) is compared to the calculated via DFT $G(P, T \sim 0 K)$ (circles). This exemplifies the limited range of applicability for the SG model. The solid-line is the fit to the DFT calculation using Eqn. 3.

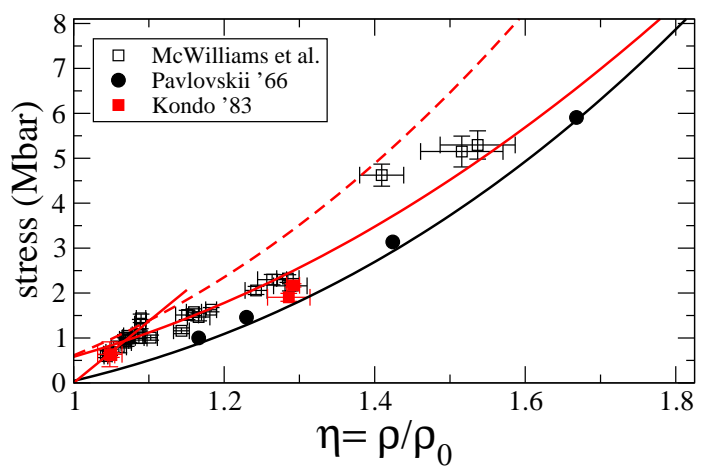

FIGURE 2. The Hugoniot stress versus compression $\eta$ comparing the theoretical estimates to the experimental data: (solid circles)[23], (solid squares)[24], and (open squares)[25]. The dashed-line is an empirically parametrized SG model and the solid-red-line is the ISG model based on DFT. Both are added to the hydrostat from the calculated EOS[26].

ison to the 1-dimensional particle velocity histories presented below. An estimate for $Y_{0} \approx 0.35 \mathrm{Mbar}$ analyzing silicon and germanium has been given[3]. Shock experiments have been performed as well up to $0.5 \mathrm{Mbar}$ where the Hugoniot state was determined still to be elastic[27]. Other shock experiments give an estimate of $Y_{0} \approx 0.63 \mathrm{Mbar}$ [24]. From diamond-anvil compression (DAC) $Y_{0}$ seems to be in the range of $1.3-1.4 \mathrm{Mbar}$ at $P \approx 2$ and at ambient temperatures[4]. Calculations give the ideal yield to be $Y_{0}<200 \mathrm{Mbar}[5]$, thereby giving an upper limit. From the data of McWilliams[25] (Fig. 2), we obtain
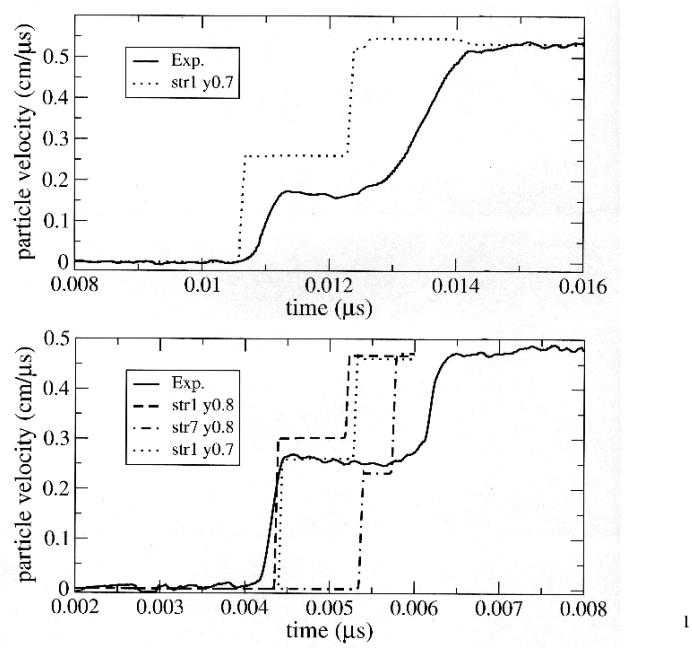

FIGURE 3. Top: A comparison between experimental (solid line) and simulated $U_{f}$ is given. The experimental single-crystal sample is oriented in $\langle 100\rangle$ and is $84 \mu \mathrm{m}$ thick. The SG model (dashed line) and ISG (dot-dashed line) both with $Y_{0}=0.8 \mathrm{Mbar}$ have an elastic wave amplitude either above or below respectively to the experimental wave. By varying the $Y_{0}$ to 0.7 Mbar with the SG model better agreement is obtained with the experiment, however the second wave is running significantly faster than experiment. Bottom: The experiment (solid line) with single-crystal oriented in $\langle 111\rangle$ and with thickness of $202 \mu \mathrm{m}$ is compared to a simulation using the SG model and $Y_{0}=0.7$ Mbar. The two wave structure in this case compares much less favorably (see text).

$Y_{0} \approx 0.7-0.8$ Mbar in comparison to the theoretical hydrostat[26]. Considering that it is above the dynamic and just below the static observations and considering thermal softening along the Hugoniot as well, our value of $Y_{0}$ is very reasonable.

In the following we examine the parametrization from the previous section through 1-dimensional planar shock simulations of polycrystalline diamond comparing with experimental free-surface particle velocities $U_{f}$ which are single-crystal with a given orientation[25]. Though really these should not be compared, it does provide a guide to the performance of the current parametrization. In Fig. 3 we compare the simulated shock $U_{f}$ and the experimental $U_{f}$ at the free surface. The experimental $U_{f}$ indicate two wave structures, which is associated with an instability $\partial \sigma / \partial \rho<0$, due to an elastic-plastic (e-p) transition in this case. In Fig. 3 we have initially varied the model from SG to ISG with $Y_{0}=0.8 \mathrm{Mbar}$, which 
brackets the elastic peak $U_{f}$. By noting that the second wave speed in SG model is slower than ISG, but still significantly faster than the experiment, then we varied the $Y_{0}$ to understand any sensitivity. By fixing $Y_{0}=0.7$ Mbar and the model to $\mathrm{SG}$, we have simulated other experiments similar to Fig. 3. From the experiments the $<111>$ crystal orientation had consistently a lower e-p transition compared to the $<100>$ and $<110>$ orientations, due the great number of slip planes available. Comparing an isotropic, polycrystalline diamond model with single-crystal data, deficiencies are expected, until more appropriate, constraining data is available. Also, even in the highest pressures (not shown) of $\approx 4.5 \mathrm{Mbar}$, the experiments indicate a two wave structure with a small time difference between the first and second wave, while the simulations waves are over-driven, i.e. a single shock wave. This may be due the single versus polycrystalline effects, like the shear modulus averaging.

In conclusion, an initial parametrization of two strength models, SG and ISG, have been performed based upon robust DFT calculations of the elastic moduli up to 10 Mbar and empirical observation for $Y_{0}$. With this, we have performed hydrodynamic 1-D simulations of a isotropic, polycrystalline diamond and have compared them to single crystal diamond experiments as a rough indicator to the models performance. Despite the limited information, the model captures the general features of the shock wave, and is expected to be comparable for polycrystalline diamond samples.

\section{ACKNOWLEDGMENTS}

Appreciation is given toward those who have yet to publish their results: S. Mc Williams, D. Hicks, J. Eggert, P. Celliers and others. This work was performed under the auspices of the U.S. Department of Energy by the University of California Lawrence Livermore National Laboratory under contract W7405-Eng-48.

\section{REFERENCES}

1. Hemley, R. J., Mao, H. K., Shen, G., Badro, J., Gillet, P., Hanfland, M., and Häusermann, D., Science, 276, 1242 (1997)
2. Correa, A. A., Bonev, S. A., and Galli, G., PNAS, 103, 1204-1208 (2006).

3. Ruoff, A. L., J. Appl. Phys., 50, 3354 (1979).

4. Eremets, M. I., Trojan, I. A., Gwaze, P., Huth, J., Boehler, R., and Blank, V. D., Applied Physics Letters, 87, 141902 (2005).

5. Zhao, J.-J., Scandolo, S., Kohanoff, J., Chiarotti, G. L., and Tosatti, E., Applied Physics Letters, 75 , 487-488 (1999).

6. Roundy, D., and Cohen, M. L., Phys. Rev. B, 64, 212103 (2001).

7. Suzuki, T., Yonenaga, I., and Kirchner, H. O. K., Phys. Rev. Let., 75, 3470 (1995).

8. Chacham, H., and Kleiman, L., Phys. Rev. Lett., 85, 4904 (2000).

9. Renshaw, C. E., and Schulson, E. M., Nature, 412, 897 (2001)

10. Eggert, J. H., Celliers, P. W., and et al., Temperature measurements of liquid carbon hugoniot (2007), private communication.

11. Steinberg, D. J., Cochran, S. G., and Guinan, M. W., J. Appl. Phys., 51, 1498 (1980).

12. Klepeis, J., and Bales, S., Improved steinberg-guinan model, private communication.

13. Hohenberg, P., and Kohn, W., Phys. Rev., 136, B864-B871 (1964).

14. Kohn, W., and Sham, L. J., Phys. Rev., 140, A1133-A1138 (1965).

15. Gonze, X. et al.., Z. Kristallogr., 220, 558-562 (2005).

16. Troullier, N., and Martins, J. L., Phys. Rev. B, $\mathbf{4 3}$ 8861-8869 (1991).

17. Perdew, J. P., Burke, K., and Ernzerhof, M., Phys. Rev. Lett., 77, 3865-3868 (1996).

18. Monkhorst, H. J., and Pack, J. D., Phys. Rev. B, 13 5188-5192 (1976).

19. Mounet, N., and Marzari, N., Phys. Rev. B, 71 205214-1 (2005).

20. Orlikowski, D., Söderlind, P., and Moriarty, J. A., Phys. Rev. B, 74, 054109 (2006).

21. McSkimin, H. J., and Andreatch, P., J. Appl. Phys., 43, 2944 (1972).

22. Grimsditch, M. H., and Ramdas, A. K., Phys. Rev. B, 11, 3139 (1975).

23. Pvalovskii, M. N., Soviet Phys. Solid State, 13, 741 (1971).

24. Kondo, K., and Ahrens, T. J., Geophys. Res. Let., 10, 281 (1983).

25. McWilliams, R. S., Hicks, D., Eggert, J. H., Celliers, P. W., and et al., Laser shock experiments on diamond: free surface particle velocity histories (2007), private communication.

26. Benedict, L., Equation of state for high pressure carbon, private communication.

27. Boteler, J. M., and Gupta, Y. M., Phys. Rev. B, 66, 014107 (2002). 